\title{
Desarrollo sostenible, medio ambiente y economía en el sector forestal
}

\author{
Raymundo Dávalos Sotelo ${ }^{1}$ \\ Francisco Morosini Cordero ${ }^{2}$
}

\begin{abstract}
RESUMEN
Se analizan las relaciones entre economía y medio ambiente, dentro de un contexto de desarrollo sustentable o sostenible en el sector forestal de México. El argumento central es que los postulados de ambos no son necesariamente contrapuestos y se puede lograr un desarrollo armonioso, con la condición de mantener una serie de principios, particularmente, tomando en cuenta la opinión de la sociedad dentro de los planes gubernamentales. En el análisis del entorno ecológico, se destacan algunos puntos relacionados con el efecto de la producción industrial en el cambio climático global y se mencionan algunas maneras de mitigarlo. Se detallan los retos que enfrenta el sector forestal en este país, así como el impacto de la industria forestal en el desarrollo nacional. Se mencionan los planteamientos de un Plan Sectorial Forestal Estatal que podría servir de modelo a otros planes regionales o estatales. En conclusión, se vislumbra un panorama positivo para el sector forestal en México, siempre y cuando se pongan en práctica los principios del desarrollo sostenible con el apoyo de toda la sociedad.
\end{abstract}

PALABRAS CLAVE:

Economía, medio ambiente, desarrollo sostenible, reciclado, cambio climático, productos forestales.

\begin{abstract}
The relationships between economy and environment are analized within the context of sustainable development in the forest sector of México. The central argument is that the premises of both are not necessarily in opposition and that a harmonious development can be achieved, provided that a series of principles are upheld, and in particular, the opinion of all of the society is taken into consideration in the governmental plans. From an ecological point of view, some points in relation with the effects of the industrial production on the global warming are discussed and some ways of mitigating it are mentioned. The challenges that the forest sector faces in México are detailed, along with the impact of the forest industry in the national development. The propositions of a State Forest Development Plan are mentioned, arguing that this sort of plan could be a model for other States. As a conclusion, a positive outlook for the forest industry is foreseen, on the condition that the principles of sustainable development are put into practice with the support of the society as a whole.
\end{abstract}

KEY WORDS:

Economy, environment, sustainable development, recycling, climate change, forest products.

1 Productos Forestales y Conservación de Bosques. Instituto de Ecología, A.C. Apdo. Postal 63. 91000 Xalapa, Ver.c.e.: davalos@ecologia.edu.mx.

2 Facultad de Ciencias Administrativas y Sociales. Univ. Veracruzana. Xalapa, Ver. c.e.:moros4@latinmail.com Manuscrito recibido el 12 de junio del 2000. Aceptado el 2 de agosto del 2000. 


\section{INTRODUCCIÓN}

Las relaciones entre economía y protección del medio, recientemente han recibido mucha atención en el ámbito científico, estableciendo relaciones entre las causas inmediatas del deterioro ambiental con la obtención de beneficios por parte de los que aprovechan los sistemas naturales. El ambiente natural es esencial para la existencia de la humanidad y es la fuente principal para su vida y producción social. Gran parte de la sociedad se pregunta qué le sucederá al ambiente en 50 ó 100 años, en vista de la creciente presión que los sistemas productivos le están aplicando y debido a los rápidos cambios que están teniendo lugar. Esta es una pregunta difícil de responder debido a que muchos de los procesos que ocurren en la naturaleza y la sociedad y muchas de las interacciones entre ellos, son todavía un misterio para nosotros. La técnica de predecir, particularmente en el campo de la Sociología, aún no está muy desarrollada. Aunque la planeación racional ha tratado de contribuir a este aspecto. Todavía es muy difícil predecir por ejemplo qué cambios socioeconómicos ocurrirán en los países en desarrollo en 100 años y qué efectos tendrán estos cambios sobre el medio ambiente.

A la fecha conocemos muy poco acerca de los ciclos dinámicos de los procesos naturales o acerca de la rotación natural de la materia y la energía, y todavía sabemos menos acerca de cómo controlarlos. Desafortunadamente, el estudio de estas interacciones en ambientes individuales y en relación con el conjunto de la tierra, así como los medios de predecir las consecuencias de la interferencia de la humanidad en el desarrollo del paisaje, no van al mismo ritmo con los cambios que están ocurriendo en la naturaleza y la sociedad.

En vista del hecho de que la producción mundial ha alcanzado proporciones extraordinarias y que nuestro conocimiento de la naturaleza, así como nuestra habilidad de controlarla, es todavía incompleta, debemos ejercitar la mayor precaución al explotar los recursos naturales de tal manera de no perturbar el equilibrio dinámico relativo de los procesos en la esfera terrestre (Ryabchikov, 1975).

En este documento planteamos el tema de discusión de que aunque el mercado puede ayudar a resolver los problemas ambientales, es preciso tener una adecuada regulación de los procesos productivos, la cual no será posible si la mayoría de la sociedad no toma conciencia de esta necesidad. El enfoque planteado aquí tomará como caso práctico el tema de los productos forestales y el desarrollo industrial en este sector.

En este sentido se consideran los impactos del desarrollo forestal, desde un punto de vista económico, social y ambiental. El análisis no ofrece una propuesta definitiva para valorar todos los aspectos y evaluar su impacto, dado que los datos básicos con que se cuenta son incompletos y la evaluación es difícil, especialmente al valorar los apartados no comerciales. Sin embargo, representa un modelo que sugiere los aspectos que deberán tomarse en consideración.

\section{EL ENFOQUE DEL DESARROLLO SOSTENIBLE}

Los postulados de la economía y de la ecología, no se contraponen necesariamente. Es posible lograr una integración armoniosa entre ambos, con base en lo que se ha denominado como desarrollo sostenible o sustentable, el que reconoce la necesidad de auspiciar el avance económico de los países menos desarrollados, aprovechando los progresos tecnológicos de las naciones industrializadas, siempre y cuando no se rebasen ciertos umbrales de calidad ambiental. La sostenibilidad es también una 
necesidad intrínseca para el desarrollo de los países pobres, ya que es imposible que puedan salir de esa condición si continúan deteriorando sus recursos: agua, suelo y bosques. La contaminación es en sí misma un desperdicio y por lo tanto disminuye las riquezas a mediano y a largo plazo. Estos puntos de vista son válidos también para las sociedades más avanzadas, las que podrían crecer más, dentro de parámetros económicos y ecológicos a la vez, generando combustibles limpios y equipos descontaminantes, e incrementando la productividad sobre la base de tecnologías limpias (Martín, 2000).

Los antecedentes del concepto de sostenibilidad se encuentran en el famoso Informe Brundtland, llamado así por haber sido formulado por una Comisión de Juristas presidida por la primera ministra de Noruega (Brundtland, 1988).

La definición de la Comisión Brundtland es la siguiente:

"El desarrollo sostenible es el desarrollo que satisface las necesidades de la generación presente sin comprometer la capacidad de las generaciones futuras para satisfacer sus propias necesidades".

Como precedentes inmediatos de este informe están la Conferencia de Estocolmo, realizada en 1972, y entre cuyas acciones más significativas estuvo la decisión de crear una nueva institución que coordinara las actividades ambientales dentro del sistema de las Naciones Unidas, Ilamado PNUMA (Cicin-Sain, 1993), el cual produjo en 1987 el documento denominado "Perspectivas ambientales para el año 2000 y siguientes; también puede citarse la Carta Mundial de la Naturaleza adoptada en 1982 por la Asamblea General de las Naciones Unidas.

Otro antecedente lo conforma la Declaración del Consejo de Europa de 1985, la cual señala las relaciones recíprocamente benéficas entre economía y ambiente y posteriormente los Programas de Acción IV y V de la Comunidad Económica Europea que recogen la proposición de la vinculación de la dimensión ambiental con las políticas que se proponen crear las condiciones necesarias para un crecimiento económico sostenido. En particular el V Programa llamado precisamente "Hacia un desarrollo sostenible", define a este como:

"Una política y estrategia de desarrollo económico y social continuo que no vaya en detrimento del medio ambiente ni de los recursos naturales de cuya calidad depende la continuidad de la actividad y del desarrollo de los seres humanos".

Finalmente, podemos mencionar la Declaración de Río de Janeiro sobre Ambiente y Desarrollo en la cual más de 150 naciones se comprometieron a asumir el principio de la sustentabilidad. Un documento fundamental derivado de esta Conferencia es la Agenda 21, que plantea la relación entre medio ambiente y desarrollo y destaca el papel de los bosques como recurso renovable, generador de productos y servicios para el desarrollo local y nacional (CNUMAD, 1992; Morosini, 2000).

Para América Latina, fueron particularmente importantes los esfuerzos de articulación entre desarrollo y ambiente emprendidos por la CEPAL y otras instituciones. Estos esfuerzos lograron introducir el tema ambiental en los esquemas tradicionales del desarrollo económico latinoamericano y a partir de ellos se promovió la adopción de políticas ambientales. En nuestro entorno más cercano, podemos citar al Tratado de Libre Comercio de Norteamérica que en su preámbulo asienta "el compromiso de los tres países que lo conforman para promover el Desarrollo Sostenible". A nivel nacional, de manera general, nos podemos referir a los pronunciamientos del gobierno mexicano sobre la necesidad de "impulsar una política capaz de modificar hábitos 
sociales y productivos para lograr un desarrollo sustentable" (Morosini, 2000).

\section{EL DESARROLLO SOSTENIBLE DESDE LA PERSPECTIVA DEL SECTOR FORESTAL}

Para las industrias silvícolas y afines, el desarrollo sostenible se ha convertido en una de las máximas prioridades en los últimos tiempos. Para ello, debe alcanzarse un equilibrio general entre los aspectos ambientales, sociales y económicos. El reto es, por lo tanto, doble: este sector debe mejorar sus resultados medioambientales de forma continua, al tiempo que debe incrementar su competitividad, lo que le permitirá crear empleos y generar productos con valor agregado. En lo que se refiere a la normatividad medioambiental, debe incluir disposiciones previsibles y coherentes cuya aplicación sea viable desde el punto de vista económico y social, para que dicha competitividad y los empleos del sector puedan mantenerse. Es preciso que todos los participantes mantengan su compromiso para aplicar un enfoque equilibrado a los pilares de la sostenibilidad (González, 2000).

El manejo sostenible de los bosques es vital para mantener todas las funciones ecológicas, sociales y económicas de los recursos forestales, entre otras la necesidad de garantizar un suministro constante de materias primas a precios competitivos. El mayor grado de conciencia y la preocupación ante los temas ambientales requieren unas políticas eficaces y coordinadas, en los niveles adecuados, sobre bosques, utilización del suelo, medio ambiente, industria, energía, ingresos fiscales y comercio exterior.

Hasta hace pocos años el manejo silvícola, el abasto y la industria de la madera en la mayor parte de México, mantenían modelos de producción anticuados y poco competitivos, expuestos a los embates de las crisis económicas y a las nuevas condiciones del mercado. Hasta la fecha, la reforestación no ha logrado revertir el cambio de uso del suelo, de lo agropecuario a lo forestal y tampoco equilibra el desgaste de la tala clandestina. Al mismo tiempo, las escasas opciones productivas para la población rural, ocasionadas a su vez por la falta de capital, capacitación y tecnología fomentan la destrucción de los últimos relictos de vegetación y por ende de su biodiversidad.

Para promover el desarrollo de las zonas boscosas del país, es preciso demostrar y visualizar a escala nacional y regional que el manejo sostenible de los bosques se puede convertir en un vector importante de cambio social político y ambiental. En este marco la formulación y aplicación de programas forestales nacionales 0 estatales constituyen un elemento esencial de la gestión sostenible de la estrategia forestal.

Dentro de este orden de ideas, y a nivel local, cabe mencionar que el Gobierno del Estado de Veracruz ha elaborado un Plan Sectorial Forestal con un amplio horizonte (1996-2034), donde el tema del desarrollo sustentable merece atención especial. En palabras de los autores de este Plan, una de sus motivaciones principales se expresa de la siguiente manera:

"Es por esto que escuchamos es reclamo de la sociedad en general por impulsar el desarrollo sustentable, lo que ha propiciado que las autoridades y la sociedad civil organizada busquen nuevas alternativas de atención al sector forestal" (Plan Sectorial, 1996).

El Plan Forestal del Estado de Veracruz busca dar una respuesta real y a la altura de la problemática que lo origina. Fue concebido como un instrumento de negociación con todas las posibles fuentes de financiamiento para que lo reconozcan como compromiso para convertir el 
problema forestal en una oportunidad de desarrollo sustentable.

Por lo que respecta a México en su conjunto, en materia forestal se debe propiciar el uso racional de los bosques, dado que si no se utilizan económicamente éstos están destinados a morir. En México las condiciones para el desarrollo forestal no han sido propicias. La carencia de una cultura forestal, ha dado como resultado el mal aprovechamiento de los recursos forestales. Sin embargo, las instituciones han emprendido un esfuerzo de planeación a largo plazo que vale la pena recalcar, puesto que ha generado resultados importantes.

Según Kokkarinen (citado por Rodríguez, 2000), al bosque se le debe considerar como negocio, ya que de lo contrario no podrá aportar beneficios. En su concepto los bosques son de suma importancia y debe vérseles como la primera etapa de la cadena productiva de la industria forestal. Los actores fundamentales relacionados con las actividades de mejoramiento y aprovechamiento de los recursos naturales, y en particular de los bosques, son los beneficiarios de estas actividades que incluyen a los campesinos que trabajan en el bosque y a los demás integrantes de la cadena de producción hasta llegar al sector industrial.

En opinión de este especialista la deforestación es causada por la ganadería, la agricultura y problemas derivados de la burocracia del sector, así como por efectos naturales tales como incendios y plagas. Un problema fundamental relacionado con la producción anual de madera es la enorme cantidad de tala clandestina que algunos autores consideran equivale a la producción nacional legalmente registrada (Kokkarinen, 2000; Plan Sectorial, 1996).

\section{EL NECESARIO REAJUSTE DE LA ECONOMÍA DE MERCADO}

El enfoque aquí propugnado, exige una reconsideración adecuada de los mecanismos, hoy predominantes del juego de la oferta y la demanda, lo que afecta tanto a los teóricos como a los actores de la economía. Desde una primera perspectiva, se ha observado que "un país podría agotar totalmente sus recursos sin que su Producto Interno Bruto (PIB) se vea afectado" (Cairncross, sin fecha). Según este mismo autor "resulta fácil ponerle precio a un árbol si se considera únicamente como madera. Pero este precio de ninguna manera reflejará su valor como mecanismo de prevención de la erosión del suelo, o como lugar de pájaros e insectos o como almacén de un dióxido de carbono que de otro modo podría añadirse a los gases responsables del efecto invernadero en la atmósfera". Esto es, se debe valorar también al bosque por los servicios ambientales invaluables que presta.

En opinión de Morosini (2000), el concepto de desarrollo sustentable adolece de deficiencias conceptuales tanto desde la perspectiva económica (dificultades para valorizar económicamente a la naturaleza, para determinar precios de los componentes del medio, para establecer sistemas contables económico-ambientales, para re-elaborar las políticas fiscales, para controlar las externalidades con instrumentos y mecanismos eficaces, entre muchas otras), como desde la ambiental (la falta de un marco conceptual adecuado para el manejo integrado de recursos naturales, la incomprensión de las bases ecológicas de las tecnologías tradicionales y modernas), o desde sus interacciones (falta de conocimientos precisos entre ecosistemas y población, entre pobreza y deterioro ambiental, por ejemplo). Además de estas deficiencias conceptuales, podemos añadirle la falta de operatividad del concepto, ya que a la fecha la discusión ha sido más retórica que de propuestas. 


\section{EL CASO DEL CAMBIO CLIMÁTICO}

Los productos de la madera y del papel, especialmente los más duraderos, desempeñan un papel significativo en el cambio climático, ya que amplían el efecto de sumideros de carbono de los bosques. La utilización creciente de productos de madera contribuye tanto a estimular el crecimiento de los árboles y la captura del carbono como incrementar el efecto de acumulación del carbono al mismo tiempo que se reducen las emisiones gracias a la sustitución de materiales (González, 2000).

No cabe ninguna duda de que la mayor amenaza que gravita sobre la tierra es el incremento de los gases que provocan el efecto invernadero. Sin embargo, hasta la fecha no se han adoptado previsiones que tengan efecto tangible en la limitación de las emisiones. Lo que el Protocolo de Kioto en 1987 propuso como compromiso fue la reducción de la media de las emisiones de gases invernadero en el período 2008-2012 en un $5.2 \%$ con respecto a las emisiones de 1990, lo que supondría un $8 \%$ de reducción para la Unión Europea, 7\% para Estados Unidos y $6 \%$ para Japón. Pero ni siquiera en estos modestos propósitos se ha avanzado y la Cumbre de Buenos Aires de 1998 significó un rotundo fracaso, aplazándose hasta el año 2000 la introducción de los mecanismos reductores (Martín, 2000).

Las industrias silvícolas y afines en los países desarrollados son consumidoras de energía de niveles medios a altos. Dados los avances significativos conseguidos en materia de eficiencia energética, las posibilidades de mejora son reducidas. Debe fomentarse la recuperación de energía mediante la incineración de residuos de madera y de papel, que son recursos energéticos renovables, lo que permitiría reducir el recurso a los combustibles fósiles. Por ejemplo en la Unión Europea se han propuesto aumentar el porcentaje de energías renovables de un $5 \%$ a un $12 \%$, para el año 2010 . Se prevé que el mayor incremento proceda de la biomasa, es decir, de los bosques y de la agricultura. Dado que la madera es una materia prima común de las industrias forestales y afines, la necesidad de garantizar un suministro permanente $y$ confiable es de la mayor importancia. (González, 2000).

\section{MANEJO DE RESIDUOS Y RECICLADO DE PRODUCTOS}

El desarrollo sostenible también supone una mayor recuperación de los productos de madera, papel y cartón usados y a la postre, una menor cantidad de residuos. La normativa sobre manejo de residuos constituye un desafío especial en un sector que en algunos países ya se dedica a recuperar los residuos de la madera y del papel, para obtener materia prima secundaria (fibra) o energía derivada de fuentes renovables.

En algunas partes del mundo, sobre todo en Europa y Norte América, ya se han registrado avances significativos en lo que se refiere a la reducción de las emisiones, gracias a la mejora de las técnicas y de los procedimientos aplicados. Sin embargo, aún en estos países sigue pendiente el desafío de aplicar las mejores prácticas en los distintos sectores, especialmente mediante la aplicación de las mejores técnicas disponibles para cada sector, especialmente para los sectores de la pulpa y del papel. En este ámbito falta por determinar la forma más conveniente (residuos líquidos, gaseosos, sólidos), para mejorar los resultados en materia de emisiones (González, 2000).

\section{DESAFÍOS QUE DEBE ENFRENTAR EL SECTOR FORESTAL EN MÉXICO}

México cuenta con los recursos físicos y humanos necesarios para convertir la actividad forestal en una alternativa de 
desarrollo preponderante. Sin lugar a dudas, buena parte del potencial económico maderero, no se finca en el aprovechamiento de los bosques y selvas, sino en el establecimiento de plantaciones forestales con diferentes objetivos. El avance de la actividad forestal a través de un fuerte programa de plantaciones restará presión a los bosques y selvas, y permitirá que estos recursos se conserven, fomentando así su potencial aún desconocido.

El desarrollo industrial, la generación de empleos y la conservación de los relictos de vegetación, serán consecuencias lógicas del desarrollo forestal. Los programas forestales deberán atender las necesidades mas apremiantes de tal manera que se puedan resolver los principales problemas forestales del país, aprovechando las potencialidades y definiendo la nueva función del Estado.

La participación de las instituciones del gobierno, ha quedado plasmada en las leyes y normas que se han emitido; sin embargo, generalmente no se han tomado en cuenta las opiniones del grupo de poseedores y propietarios del recurso forestal y de los habitantes de las zonas forestales, que superan los 17 millones de habitantes. Esta situación condujo a la necesidad de un cambio en la participación y en las estrategias, para que una vez identificadas las necesidades, demandas y aspiraciones de las comunidades rurales, los gobiernos federal, estatal y municipal, puedan hacer planteamientos legales y normativos en general, que estén en sentido paralelo con el desarrollo integral de los campesinos dedicados a la actividad forestal quienes tienen el derecho de obtener los beneficios del bosque, que como recurso natural es eternamente renovable si se somete a un manejo técnico-científico, sin trabas burocráticas que lo obstaculicen o lo eviten, como ha sucedido hasta la fecha.
Todas las leyes y normas que se han expedido o se vayan a expedir, serán letra muerta si no se ligan los problemas socioeconómicos y los propios intereses de los dueños y poseedores de los bosques y selvas, con el interés decidido de las instituciones gubernamentales, dispuestas a someter los recursos forestales del país a un programa agresivo para manejarlo científicamente, con la firme decisión política de generar la riqueza permanente que significa el aprovechamiento sostenido, para beneficio de los pobladores.

Los desafíos más importantes que debe afrontar en los próximos años el sector forestal en relación con el desarrollo sostenible y la protección del medio ambiente son:

C fomentar el manejo sostenible de los bosques

C contribuir de forma activa y competente a que la legislación y los instrumentos voluntarios sobre energía, sean coherentes, previsibles y sostenibles desde el punto de vista de la competitividad

C garantizar el suministro de energía con precios competitivos

$C$ garantizar un suministro permanente de madera con precios competitivos de conformidad con la política de producción de energía procedente de fuentes renovables

C mejorar los resultados ambientales y energéticos del sector

Cinformar sobre los resultados ambientales recurriendo a métodos de medición aprobados

\section{IMPACTO ECONÓMICO Y ECOLÓGICO DEL SECTOR FORESTAL EN MÉXICO}

Para hacer frente a estos desafíos es necesario que la legislación y las medidas adoptadas se basen en una evaluación científica adecuada, así como en los correspondientes análisis de costos y 
beneficios. México tiene la condiciones ideales para el buen funcionamiento de bosques y plantaciones comerciales, pero deben vencerse los enemigos del bosque anteriormente mencionados, entre los cuales la rígida burocracia no es el menor (Kokkarinen, 2000).

Por ser un ejemplo importante de planeación a nivel nacional y por tratarse de una entidad seleccionada como modelo por las autoridades federales, en los siguientes párrafos se recogen algunos de los planteamientos que se consideran más consistentes del Plan Sectorial Forestal del Estado de Veracruz, que bien podrían extrapolarse a todo el país.

Según este Plan, en algunas regiones del país, la actividad forestal puede ser más rentable en plantaciones. EI aprovechamiento de los bosque templados, selvas y las plantaciones templadas y de clima cálido-húmedo comerciales también tiene perspectivas positivas. Por otro lado, el objetivo de las plantaciones ecológicas es la conservación, no los beneficios económicos. Por lo tanto, no se puede criticar la rentabilidad negativa de estas áreas.

La posibilidad de establecimiento de industria forestal dependerá de la oferta de la materia prima estimada. Cuando ésta sea importante, se podrá expandir la industria. La importancia socio-económica de los talleres y fábricas de procesamiento secundario puede ser aún mayor que la de aserraderos. También se pueden dar las condiciones para los procesos en una planta industrial procesadora de celulósicos.

La producción no maderable puede tener un impacto socio-económico considerable. Este tipo de producción ofrecería ingresos para el propietario del bosque, antes de la producción maderable $y$, por tanto, aumentaría la rentabilidad de la producción.
Un efecto positivo del desarrollo forestal es que la infraestructura de comunicaciones y transportes se amplía. Como consecuencia, las comunidades estarían menos aisladas, y existirían más programas de desarrollo. La planta productiva crecería. Al generarse empleo la población se arraigaría a sus comunidades. Los ingresos que generaran los aprovechamientos repercutirían en el incremento de la derrama económica en las regiones forestales. Aumentaría la calidad de vida de los moradores de las zonas forestales.

Con la integración de nuevas áreas forestales al manejo se revertiría la tendencia hacia la deforestación. Se deberán reforestar incluso áreas degradadas donde la producción agrícola dejó de ser una alternativa viable. La cultura forestal aumentaría y se generaría un interés por manejar el recurso forestal buscando su permanencia a largo plazo. La reforestación cambiaría las áreas no productivas a bosques homogéneos.

Mediante la incorporación al manejo de más hectáreas de bosques y selvas, se garantizaría la conservación de la vegetación de esas áreas. Esta situación tendría un efecto directo sobre la captación de agua y la conservación del suelo. Áreas importantes de plantaciones serían para recuperación de áreas degradadas en las cuencas altas y para fijación de dunas en las partes bajas. El mejoramiento de la cantidad y calidad de agua aumentaría directamente el nivel de salud pública.

La conservación de la biodiversidad se incrementaría pues con la incorporación de nuevas superficies de bosque y selvas naturales al manejo, se estarían seleccionando los mejores ejemplares de la flora mejorando la calidad genética de las poblaciones naturales. Hasta aquí, el análisis del Plan Sectorial. 
La contribución del sector forestal al desarrollo sostenible podría reforzarse con las siguientes medidas 0 ámbitos de actuación:

Cfomentar formas rentables de manejo sostenible de los bosques elaborando programas forestales nacionales 0 regionales y contribuyendo a la elaboración del marco adecuado para dicho manejo.

Cmejorar los resultados del sector en materia de medio ambiente y de energía mediante un esfuerzo permanente de investigación y desarrollo, la comparación de los resultados en estos ámbitos y la adopción de nuevas tecnologías y otras soluciones rentables.

Cmejorar el manejo de residuos mediante un enfoque general que incluya técnica rentables de reciclado y recuperación de energía, así como soluciones innovadoras para la eliminación final, encontrando los medios y la forma (líquida, gaseosa 0 sólida), más adecuada para reducir los efectos eficazmente.

Cevaluar los efectos de captura y acumulación de los productos derivados de la madera y de la recuperación de energía y evaluar la incidencia en que la utilización creciente de estos productos pueda tener un impacto positivo en el cambio climático, teniendo así mismo en cuenta las sustitución de materias más perjudiciales para el medio ambiente.

Cdeterminar, en materia de medio ambiente y de energía, ámbitos en los que pueda conseguirse valor agregado mediante instrumentos voluntarios eficaces y contribuir de forma activa y competente al proceso legislativo.

Celaborar informes sectoriales sistemáticos y completos, sobre medio ambiente y energía.

\section{CONCLUSIONES}

Si se toman en cuenta estas y otras propuestas semejantes, los bosques naturales y cultivado serán rentables y podrán ser considerados como negocios generando fuentes de ingreso con impactos en el medio rural considerable e inmediato. Si se le da un buen manejo al bosque, podemos garantizar para esta y las siguientes generaciones, un resultado satisfactorio de los siguientes temas: suministro y manejo adecuado del agua, control de la erosión, mejoramiento de la calidad del aire, aumento de la base económica y aportación de beneficios sociales.

Para lograr esto, México necesita opciones múltiples para el desarrollo forestal. La iniciativa privada como productor de madera, debe propiciar la creación de zonas maquiladoras forestales. Por su parte el sector social (ejidatarios), debe tratar a la industria como cliente, no como patrocinador.

Para acceder al desarrollo sustentable, será necesario involucrar a todos los ámbitos de gobierno, particularmente a la Secretaría de Medio Ambiente Recursos Naturales y Pesca (o lo que se convierta en el nuevo régimen), además de otras secretarías del gobierno federal relacionadas con el tema del campo y de la industria, para elaborar un verdadero plan estratégico que fomente la apertura y la descentralización de sector. La participación de la organizaciones no gubernamentales también será vital en la formulación de una total integración de la dimensión ambiental en las políticas económicas y en la toma de decisiones en cualquier actividad en los ámbitos nacional e internacional.

En el caso de las selvas tropicales, se ha propuesto que la integración de los esfuerzos conservacionistas locales con las políticas de financiamiento de los organismos públicos sería una manera de tener un enfoque mas eficiente para el uso sustentable de ellas (Aguilar, 1998). Uno de los mayores retos para la conservación de las selvas consiste en enfocar estos esfuerzos conservacionistas de manera 
conjunta, dirigiéndolos hacia el aprovechamiento sostenible, la definición de políticas de financiamiento de las instituciones gubernamentales y la educación, difusión y capacitación para el desarrollo sostenible de los pobladores.

En opinión de Aguilar (1998), las oportunidades que brindan las selvas, potreros y acahuales remanentes son múltiples. Para revertir las situaciones desfavorables es necesario recopilar información de tipo social, económica y ecológica y generar una base de datos. De esta manera se puede tener una visión mas precisa de las necesidades y expectativas de los grupos humanos presentes en las regiones forestales, para tratar de hacerlas compatibles con los objetivos de conservación de la diversidad biológica. Otras opciones de explotación racional de productos alternativos agroforestales también deben ser exploradas.

\section{REFERENCIAS}

Aguilar R., S. 1998. Las selvas de Uxpanapa: el último bastión. Pronatura, Primavera 1998, No.2: 46-51.

Brundtland, G.H. 1988. Nuestro futuro común. Comisión Mundial del Medio Ambiente y del Desarrollo. Ed. Alianza, Madrid.

Cairncross, F. s/f. Las cuentas de la tierra, Acento Editorial, Madrid.

Cicin-Sain, B y R.W. Knecht. 1993. Implications of the earth summit for ocean and coastal governance. Ocean Development and International Law, Vol.24:323-353

CNUMAD. 1992. Declaración de Río de Janeiro sobre Ambiente y Desarrollo.

González, M.A. 2000. Desarrollo sostenible, medio ambiente, y energía. Boletín de información técnica AITIM, 204, marzo-abril 2000: 56-62 p.

Martín M., R. 2000. La revolución ambiental pendiente. Revista Mexicana de Legislación Ambiental. Año 1 No. 2, enero-abril 2000: 31-44 p.

Morosini C., F. 2000. Desarrollo sostenible o sustentable y economía. Universidad Veracruzana, Instituto de Genética Forestal, Apuntes para el Seminario: Manejo Integral y Desarrollo Sustentable de los Bosques.

Plan Sectorial Forestal del Estado de Veracruz (1996-2034). 1996. Documento extenso. Gobierno del Estado de Veracruz, Secretaría de Medio Ambiente, Recursos Naturales y Pesca. $159 \mathrm{p}$.

Rodríguez M, J.L. 2000. Sector forestal en México. ¿Alternativa económica o catástrofe ecológica? Moblaje. Año 1 No. 5, abril 2000: 66-68.

Ryabchikov, A.1975. The changing face of earth. Progress Publishers, Moscú. 